\title{
Echocardiography: An Important Tool for Diagnosis, Guidance of Intervention and Post- Interventional Follow Up of the Congenital and Structural Heart Diseases
}

\author{
NNF Begum¹, AKM Razzaque ${ }^{2}$, MM Rahman $^{3}$ \\ ${ }^{1}$ Deptt. of Paediatric Cardiology, Combined Military Hospital, Dhaka; ${ }^{2}$ Deptt. of Thoracic Surgery, \\ IDCH, Dhaka; Deptt. of Cardiology, ${ }^{3}$ Comilla Medical College, Comilla.
}

(Cardiovasc. j. 2011; 4(1): 67-74)

\begin{abstract}
Definition of Echocardiogram:
An Echocardiogram, often referred to in the medical community as a cardiac ultrasound or simply an Echo, is a sonogram of the heart. It uses standard ultrasound techniques to image twodimensional slices of the heart. The latest ultrasound system can produce 3-D real time image. High-frequency sound waves inaudible to the human ear are sent from the probe and directed towards the heart, part of which bounces back (echoes) to the probe and is interpreted by the ultrasound machine into images, blood flow within the beating heart. The images are than displayed on the monitor and recorded on disk for future review. This test is performed either by cardiac sonographers, cardiac physiologists or doctors trained in cardiology.1-3
\end{abstract}

\section{Purpose:}

Echocardiography is one of the most widely used test for diagnosis of various heart diseases. In addition to create two or three dimensional picture of the heart and vessels, it can also produce accurate assessment of the velocity of blood and cardiac tissue at any arbitrary point using pulse or continuous wave Doppler ultrasound. So this test is helpful for assessing the size of the heart chambers and walls, heart muscle function, heart valve function, blood clots or masses in the heart, fluid around the heart, presence of holes or defects between the heart chamber or vessels, anatomical relation ship of various heart chamber and loops, abnormalities of blood flow within the heart etc. Doppler echo is helpful for assessment of cardiac valve areas and function, any abnormal communication between left and right heart, any leaking of blood through valves (regurgitation) and calculation of cardiac output, ejection fraction, E/ A ratio etc. ${ }^{3}$

\section{Recent uses:}

1. Assessment of patient for selection of various interventions like device closure of septal defects, ductus arteriosus, closure of left atrial appendage, balloon valvoplasty, angioplasty etc.

2. Guidance of interventional procedure like device closure, left atrial appendage closure, trans septal puncture, balloon atrial septostomy, strenting of duct and vessels etc.

3. Per operative assessment of operative procedure like ASD, VSD closure, Tetralogy of Fallot repair, pulmonary artery banding, Fontan operation etc.

Types of echocardiography:

1. Transthoracic Echocardiogram (TTE) - This is a test which takes a picture of the heart with the use of a transducer placed on the chest wall of the patient. This test is highly specific for diagnosing congenital heart disease, size of heart chambers, walls, cardiac functions, clots or masses in the heart and others.

2. Transoesophageal Echocardiography (TEE) As opposed to the standard echocardiogram, in TEE the transducer is placed in the esophagus directly behind the heart. In addition to use by cardiologist, TEE can be performed

Address correspondence: Dr Nurun Nahar Fatema Begum, Paediatric Cardiologist, Lab Aid Cardiac Hospital, Dhaka, Bangladesh. e-mail- colfatema@hotmail.com 
by a cardiac anaesthesiologist to evaluate, diagnose and tract patient in pre-operative period. ${ }^{3,4}$

3. Intracardiac Echocardiography (ICE) - ICE is an imaging technique that is becoming increasingly available as an alternative to TEE to guide percutaneous interventional procedure. The physics of ICE are same that are used for all applications of ultrasound. The images can be displayed in M-mode, B-mode with Doppler effects and as three-dimensional reconstruction. In eletrophysiological interventional procedure, conventional ICE was used to guide the anatomic placement of the ablation catheter and to assess cathetertissue contact. Immediate identification of complications and reduction in fluoroscopy time are other remarkable benefits of direct endocardial visualization doing radiofrequency catheter ablation. With $9 \mathrm{MHz}$ ICE, detail identification of normal and abnormal structures and cardiac anatomy can be visualized with improved imaging depth. However, all ICE devices are not steerable and must be guided by a wire. Early experience with ICE suggested that this technique could evolve as a clinically useful method with diagnostic, monitoring and guidance applications possibly leading to the conversion of catheterization laboratory into integrated imaging, monitoring and therapeutic stations. Currently ICE is used in many center for guiding $\mathrm{PFO}$ closure, ASD device closure and trans septal puncture procedure. It is an important tool for guiding transseptal puncture during ectrophysiological mapping and ablation procedure. It is also available or currently under development to guide other noncoronary interventions like percutaneaus placement of cardiac valves, closures devices etc. Intracardiac echocardiography can be use in EP laboratory during ablation procedure for visualization of pulmonary veins, location of atrial-venal junction and assurance of the ablation catheter tip location within pulmonary vein antrum. ${ }^{5-15}$

4. Fetal echocardiography: Here probe is placed over the mothers abdominal wall and directed towards fetal heart. This test evaluates the babies heart by using sound waves. This procedures provides a valuable measures to better understand intrauterine growth and development of the heart and great vessels. The prenatal diagnosis of structural heart disease and the physiological evaluation of fetal arrhythmias are perhaps the most important insights provided by this techniques. There are several potential indications for doing fetal echocardiography. Evaluation of the heart in the setting of retarded fetal growth or fetal distress is often recommended. Whenever the extracardiac anomalies are detected during fetal examination by ultrasound, cardiac assessment is indicated. Presence of chromosomal anomaly detected by amniocentesis is another indication for fetal echocardiography. ${ }^{1}$

5. Stress echocardiography -Stress echocardiography is a test that use ultrasound imaging to determine the heart muscle response to stress.

6. Contrast echocardiography - A small amount of liquid is injected through an IV cannula to the inside of heart to confirm right to left shunt through patent foramen ovalae, presence of atrial septal defect, left superior vena cava etc.

\section{Basic techniques used for echocardiography:}

Two important factors are considered in echocardiographic examination:

(1) Imaging and (2) Doppler studies

Images are two dimensional, three dimensional or real time four dimensional or M-mode of two dimensional display. Doppler studies are continuous wave Doppler, pulsed Doppler and color flow Doppler. ${ }^{1-5}$

A. M-mode imaging: It allows selection of an M-line form of the two-dimensional image for subsequent M-mode display. In pediatric patients, this images are used for measurement of various chamber size, ventricular pumping function, wall thickness etc. This is a single dimension image of the heart.

B. Two dimensional imaging: Two dimensional echo is capable of displaying a 
cross-sectional "Slice" of the beating hearts, including the chambers, valves and the major blood vessels that exit from the heart. It can give idea about size of the heart chambers, dimension of the cavity, wall thickness, presence of any congenital defects or hole, anatomical study of structures with concordance or discordance of chamber connection, pumping function of the heart etc.

C. 3D-4D Echocardiography: Transthoracic and transoesophageal echocardiography are currently able to provide $3 \mathrm{D}$ reconstruction of image as well as a real time (4D) volumetric rendering of the heart. Live 3D echo is likely beneficial in cardiac surgeons pre and post surgical planning by giving a complete accurate perspective of anatomical relationship and detecting abnormalities before surgery begins. Same is true for interventional procedures in catheterization laboratory, thus help in reducing fluoroscopy and procedure time.

D. Doppler ultrasound: Three basic types of ultrasound technology are currently available. Continuous wave (CW) doppler, pulsed wave (PW) doppler and color flow Doppler. Each technique is complementary to other techniques. CW and PW Doppler applies only to system that allow simultaneous imaging and Doppler. They can measure the velocity on sample volume. This can be measured on frozen image or in real time. Color flow Doppler can evaluate the flow of blood within the heart and vessels. This helps detection of abnormal communication inside heart, stenosis at any level or regurgitant jet .

Echocardiography for diagnostic purpose and selection of patient for intervention and surgery:

The role of echocardiography continues to play an important role in the diagnosis and assessment of response to therapy of many cardiac conditions. One of the strengths of echocardiography is that it can be brought to the patients bed side easily. Infact, features of ultrasound machines such as 2D, 3D and transoesophageal echo, spectral and colour Doppler imaging, strain rate imaging and even intracardiac imaging can be performed on devices that are increasingly portable. Many interventional procedure in the catheterization laboratory require imaging of the heart in a manner different from fluoroscopy so that the position of the devices can be optimized. By virtue of its ease of use, lack of radiation and portability, it has come to play a key role in selecting the patients for and guiding the procedure. Transthoracic or transoesophageal echocardiogram with two dimensional and three dimensional images played an important role in selecting patient for all interventions as well as surgery. For these, some criteria's are followed by cardiac centers to select their patients. ${ }^{16-33}$

These are:

A. Atrial Septal Defect (ASD)

a. Only secundum type.

b. Rim all around the defect should be more than $7 \mathrm{~mm}$ (20\% of circumference).

c. Not associated with other defects which can not be treated non-surgically.

d. Not associated with Eisenmenger syndrome.

B. Ventricular Septal Defect (VSD)

a. All muscular VSD's.

b. Perimembranous VSD without any inlet or outlet extension.

c. Perimembranous VSD without any aortic regurgitation or aortic valve prolapse.

d. Distance of aortic valve from VSD margin should be more than $3 \mathrm{~mm}$.

e. Not associated with arrhythmias, Eisenmenger syndrome.

C. Patent Ductus Arteriosus (PDA)

a. Almost all PDA's are amenable to intervention.

b. Some large tubular PDA without any pocket in aortic side are excluded.

c. Eisenmenger syndromes are also contraindication.

D. Aortic valve stenosis

a. Peak pressure gradient $>80 \mathrm{~mm} \mathrm{Hg}$.

b. Not associated with aortic regurgitation.

c. Dysplastic valve is a contraindication.

E. Pulmonary valve stenosis

a. Peak pressure gradient $>60 \mathrm{~mm} \mathrm{Hg}$.

b. Not associated with infundibular stenosis.

c. Valve not dysplastic. 
F. Percutaneous pulmonary valve implantation (In postoperative cases with RV-PA conduit).

a. Size of RV to PA conduit more than $16 \mathrm{~mm}$.

b. Age more than 05 years.

c. Weight more than $30 \mathrm{Kg}$.

d. Peak pressure gradient $>35 \mathrm{~mm} \mathrm{Hg}$.

e. More than moderate PR.

G. Coarctation of aorta

a. Pressure gradient across coarct segment $>20 \mathrm{~mm} \mathrm{Hg}$.

b. Descrete coarctation.

c. Associated with systemic hypertension.

H. Left atrial appendage closure (Indicated for patient with non valvular atrial fibrillation as more than $90 \%$ of thrombi originate in left atrial appendage)

Key points to be examined by echocardiography:

a. Number of the lobes.

b. Exclusion of presence of thrombus inside appendage.

c. Sizing of the mouth of the appendage (from multiple view).

d. Assessment of adjacent structure eg. left anterior descending artery and great cardiac vein.

I. Percutaneous Transcatheter Mitral Commissurotomy (PTMC). Echo assessment of papillary muscle, MV leaflets, chordae tendinae and annulus should be done thoroughly.

a. Mitral valve area $<1.5 \mathrm{~cm}^{2}$.

b. Symptomatic patient.

c. Mobile valve.

d. Leaflet thickness-acceptable.

e. No Subvalvar involvement.

Contraindications of PTMC:

a) Presence of LA thrombus.

b) MR grade II

c) Calcification of valve.

d) Atrial fibrillation.

e) Recent history of thromboembolism.
Echo score for PTMC
a) Score $<8$
acceptable.
b) Score $>8$ but $<12$
can be done.
c) Score $>12$
should go for surgery.

J. Transcatheter Aortic valve implantation (TAVI)

It can be performed in good surgical candidates with an expectation of an excellent outcome. However surgery in patient with comorbid condition may be associated with significant mortality and morbidity. In some such patient percutaneous aortic valve replacement may offer double benefit while avoiding many of the problems associated with sternotomy, aortotomy and cardio pulmonary bypass. ${ }^{5-7}$

K. Stenting of Coarctation of aorta

A study was conducted among 40 institution of united states and all of them consider stenting native or recurrent coarctation of the aorta, regardless of the severity of narrowing. The majority (95\%) would consider stent placement as the primary treatment in patient $>30 \mathrm{~kg}$ and balloon angioplasty the treatment of choice in patients $<30 \mathrm{~kg}$. No institution consider stenting in infants so far unless surgery is contraindicated. ${ }^{8}$

L. Closure of patent foramen ovale (PFO)

a. Assessment of right atrial opening.

b. Assessment of left atrial opening.

c. Assessment of middle region of overlapping to select the type of devices.

PFO should be closed in a patient before doing any second cerebrovascular accident (CVA). PFO, along with atrial septal aneurysm (ASA), has been implicated as a risk factor for cryptogemic stroke. In such cases TEE has demonstrated worm-like echogenic structures straddling the atrial septam through PFO. ${ }^{9}$

M. Mitra clip for mitral regurgitation

The E-valve mitral valve repair procedure is a novel approach for leaflet repair for mitral regurgitation. The procedure is performed via transseptal catheterization under fluoroscopy and TEE guide. $\mathrm{E}$ valve mitral clip grasp the mitral leaflet and create a double orifice, with resultant better approximation of the mitral leaflets and diminution 
of the degree of MR. ${ }^{10-14}$ Some of the diseases cannot be treated by intervention and they are the candidates for surgical correction.

Special diagnostic test by echocardiography

Transthoracic and transoesopheageal echocardiography has been used for confirmatory diagnosis of some doubtful conditions.

a. Contrast echocardiography is used to see the presence of thrombus in the left atrial appendage or left atrium. It is also used to confirm the presence of atrial septal defect, Persistent left superior vena cava in doubtful cases.

b. Transcarotid Doppler (TCD): This test is used to confirm the right to left shunt through patent foramen ovale. CW Doppler is placed over the carotid artery and saline is injected through the cubital vein. If there is right to left shunt, than bubble appear in the CW envelope over carotid artery. Appearance of more than 10 bubble in Doppler is positive. TCD positive patient should do his/her coagulation profile and should avoid long flight.

\section{Intraoperative / Intraprocedural guidance} provided by echocardiography.

The device closure procedure has traditionally been guided using a combination of fluoroscopic and twodimensional (2-D) trasoesophegeal (TEE) and transthoracic (TTE) imaging. Because of only two dimension, 2D is limited to detect the position of a catheter or a device relative to its surrounding structure, requiring the acquisition of multiple plane images in order to reconstruct the original setting. Recently 3D TEE is used to navigate ASD closure in infant as well as in VSD closure. In adults, image quality by TTE is often not sufficient to guide the closing procedure adequately, like appropriate deployment and position of the device with regard to the surrounding structure. So 3D TEE is very efficient and safe technique to guide interventional procedures specially device closure of PFO, ASD, VSD etc. Now a days, intracardiac echocardiography (ICE) can be used as the primary means for both selection of devices and guidance of transcatheter closure of secundum ASDs.

With ICE planes are identified with excellent resolution, providing proper measurements of fossa ovalis, from which to derive geometric assumption of the selection of an appropriately sized device. The ASO (Amplatzer Septal Occluder) waist diameter is chosen in some centre on the basis of the $r$ value $\left(r=c^{2}+p^{2}\right)$. The $r=$ radias of fossa ovalis, $\mathrm{c}$ is the foci half distance of fossa ovalis and $\mathrm{p}$ is its semilatus rectum during ASD closure.

It has been shown in some study that nonconclusive TEE results found in some children with ASD can be benefited by MR imaging for determination of defect size, rim distances to adjacent structures and venous connections. Other than device closures, most frequent interventional procedures are balloon valvotomy or angioplasty, stenting of arterial or venous stenosis, closure of intracardiac or extra cardiac communications, balloon atrial septostomy or blade atrial septectomy etc. Some structural intervention are also done like left atrial appendage closure, post myocardial infarction VSD closure, Alcohol septal oblation in HCM etc. While most of these procedures are carried out with the assistance of radiological screening, in some circumstances transoesophegeal echocardiography greatly improves the success and safety. TEE is an important part of imaging for closure of fossa ovalis ASD, occlusion of baffle fenestration following total cavopulmonary connection, closure of congenital muscular VSD, blade atrial septectomy, PTMC, and non surgical reduction of ventricular septum in hypertrophic cardiomyopathy. For transseptal puncture, echocardiography is helpful to select the location of puncture site avoidiry other adjacent structure like aorta. ${ }^{34-50}$

Some special interventional procedures:

A. Atrial septal defect (Device closure): During device closure echocardiography helps to check followings

1. Passage of catheter and wire through largest hole in case of multiple ASD or fenestration.

2. Balloon sizing of the defect to look for residual shunt or presence of any other defect in the atrial septum and to determine the stretched diameter of the defect.

3. Opening of left and right atrial disc and to see whether there is any obstruction of 
flow to pulmonary veins or superior vena cava.

4. Any regurgitation in mitral or tricuspid valve.

5. Appearance of pericardial effusion from trauma.

6. After release of device, color flow Doppler is used to look for residual shunt.

B. Ventricular septal defect (Device closure) : Suitable conditions for device closure of VSD are-
a) Muscular VSD
b) Some of the perimembranous VSD
c) Residual defect in the patch margin following cardiac surgery
d) Ischeamic defects following myocardial infarction.

A Combination of fluoroscopy and TEE is required for this procedures. During procedure TEE help in detecting followings:

a) Morphology and diameter of the defect.

b) Relation of semilunar and atrioventricular valve and tensor apparatus.

c) Provide information about the position of the wire, sheath and occluder during and after deployment.

d) Selection of the size and type of the occluder.

e) Left ventricular disc is deployed under echo guide followed by pull back of sheath and release of right ventricular disc.

\section{Left Atrial appendage closure (LAA closure)}

Transoesophegeal echocardiography is must to measure the size of the orifice, number of lobe, presence of thrombus etc. During procedure it helps in selecting size of device, type of the device, trans septal puncture and proper placement of the fixing and sealing part of the device (ACP type) and fifing of other devices.

\section{Percutaneous transcatheter mitral commissurotomy (PTMC)}

TEE is essential in small children for safe perforation of the atrial septum and for precise positioning of the balloon dilatation catheter across the mitral valve, avoiding damage to the tension apparatus.

\section{E. Alcohol septal ablation in hypertrophic cardiomyopathy:}

Procedure is monitored with TEE for precise imaging of the injection of alcohol by delineating the area with a myocardial contrast agent, injected directly through coronary catheter.

Echocardiography in monitoring, guiding and evaluating surgical repair of congenital malformation:

Trans oesophageal echocardiography is a useful tool to determine the strategies for treatment in the preoperative period and to improve the quality of surgical procedures in children with congenital heart disease. Accuracy of surgical procedure can be assessed preoperatively during VSD closure, RVOT resection, TAPVD repair and many other occasions. Residual problems or sequels are identified in immediate post operative period and some patient may go for re-intervention or return to bypass for modification of the surgical procedure. ${ }^{51-54}$

Post operative and post interventional follow up of the patient by echocardiography.

Every interventional and surgical procedures needs follow up evaluation to look for outcome, complications, residual problems etc. So every patient need to be evaluated routinely at regular interval from cardiology and cardiac surgery out patient department by ECG, Chest X-ray and most importantly by transthoracic echocardiography. If any complications or residual problem are noticed, that should be addressed properly without making delay. Some of the patient may need redo surgery. Some case of device closure patients have late device embolization in many centers in inexperienced hand, which can be identified by echocardiography. ${ }^{54,55}$

\section{Conclusion}

Two and three dimensional echocardiography using precordial or trans oesophageal or intracardiac probe has proved as an essential part of imaging in selecting patient for intervention, surgery, in safe intra operative guidance of the procedures, in effective post procedure/ operative follow up of the 
patient. In our centre we use trans thoracic / trans oesophageal guide in catheterization laboratory for every interventional procedure in children.

\section{References:}

1. Riger P. Vermilion. Basic physical principle, Technology and instrumentation. In: Rebecca Snider, Gerald Serwer, Samual Ritter, editors. Echocardiography in paediatric Heart Disease. Missouri: Mosby-year book inc; 1997. P 1-21.

2. http://en. Wikipedia.org/wiki/Transthoracic echocardiogram.

3. http;//en.wikipedia.org/wiki/transoesophageal echocardiogra.

4. Ramadan As, Stefamidis C, Ngatchow w, Lemoine O, De canniere. Esophageal stent for iatrogenic oesophageal perforation during cardiac surgery. Am Thoracic Surgery 2007;84 (3) 1034-6.

5. Susan S kim, Ziyad M Hijazi, Roberto M, Bradly PK. The use of intracardiac echocardiography and other intracardiac imaging tools to guide non coronary cardiac interventions. J Am Col Cardiol 2009;53:2117-2128.

6. Asrress KN, Mitchell AR. Intracardiac echocardiography. Heart 2009;95:327-331.

7. Rodolf C, Valentino D, Aless Andro S, Santono M, Salierno M, Giavanni G. Intracardiac echocardiography to guide transseptal catheterization for radiofrequency catheter ablation of left sided accessory pathway: two case reports. Cardiovascular Ultrasound 2004;2:20.

8. Bom N, Lanceer CT, Van Egmond FC. An ultrasonic intracardiac scanner. Ultrasonics 1972; 10: 72-76.

9. Seward J, Khanderia B, McGregor C. Transvascular and Intracardiac two dimensional echocardiography. Echocardiography 1990;7:457-464.

10. Bartel T, Caspari G, Mueller S, Erbel R. Intracardiac Echocardiography - Technology and clinical role. J Clin Basic Cardiol 2002; 5: 133.

11. Pandian NG, Hsu T. Intravascular ultrasound and intracardiac echocardiography: Concepts for future. Am J Cardiol 1992; 69: 6-17.

12. Ziyad M Hijazi, Zhong W, Qi-Ling C, Keonig P, waight D, Long R Transcatheter closure of atrial septal defects and patent foramen ovale under intracardiac echocardiography guidance; feasibility and comparisn with transoesophageal echocardiography. Cath Cardiovasc Interv 2001; 52:194-9.

13. Ziyad M Hijazi, Kalyanam shiv kumar, David J sahn. Intracardiac echocardiography during interventional and electrophysiological cardiac catheterization. Circulation 2009;119:587-596.

14. Andrew RJ, Presanna P, Jonathan T, Harald B, Neil W. The emerging role of intracardiac echocardiography Into the ICE age. $B r$ J Cardiol 2007; 14 (1):31-36.
15. http://en.wikipedia.org/wiki/fetal echocardiography.

16. Navian C Nanda. Atlas of three-Dimensional echocardiography, $1^{\text {st }}$ ed Newyork: Futura publishing company inc: 202: 129-154.

17. Bharucha T, Roman Ks, Anderson R, Vettu kattil J, Impact of multiplanner review of three dimensional echocardiographic data on management of congenital heart disease. Ann Thorac Surg 2008;86:875-881.

18. S. A Qureshi . Selection of patients with secundum Atrial septal defects for transcatheter device closure. Eur Heart Journall 2000; 21 (7): 510-511.

19. P Acar, Z saliba, P Bonhoeffer, X Aggoun, D Bonnet, D sidi, J kachaner.Influence of atrial septal defect anatomy in patient selection and assessment of closure with the cardioseal device. A three-dimensional transoesophageal echocardiographic reconstruction. Eur Heart J 2000; 21 (7): 573-581.

20. Nicholas J colins, Eric M Horlick. Atrial septal defect device closure In: Ziad M Hijazi, Ted Feldman, JP Cheatham, Horst Sievert, editors. Complications During percutaneous interventions for congenital and structural Heart Disease. UK: Informa uk Ltd; 2009. P 207-218.

21. Karim Diab, Qi-Ling Cao, Ziad M Hijazi. Device closure of ventricular septal defect (percutaneous and Hybrid) using the Amplatzer VSD devices. In: Ziad M Hijari, Ted Feldman, JP Cheathum, Horst Seivert, editors. Complications during percutaneous interventions for congenital and structural Heart disease. Uk: Informa uk Ltd; 2009. P 175-184.

22. Webb JG, Chandavimol M, Thomson CR. Percutaneous aortic valve implantation retrograde from the femoral artery. Circulation 2006; $113^{\circledR}$ : 842-50.

23. Webb JG, Pasupati S, Humphries K. Percutaneous transarterial aortic valve replacement in selected high risk patients with aortic stenosis. Circulation 2007; 116 (7): $755-63$.

24. J Balzer, S. van hall, T. Rassat, Y-C Boring, A Frankle, R-M lange et al. Feasibility safety and efficacy of real time three-dimensional echocardiography for guiding device closure of interatrial communications: Initial clinical experience and impact on radiation exposure. Eur J Echocardiography 2010; 11(6): 1-8.

25. Joseph AL, Cao QL,W Lynn, Lissa S, Lopez J, Roberto M,Hijazi M H. Feasibility of real time three-dimensional echocardiography for guidance of percutaneous Atrial Septal Defect closure. Eur J Echocardiography 2009; 10(4): 543-548.

26. Jan Balzer, Malte Kalm, Herald P Kuhl.Real time threedimensional echocardiography for guidance of noncoronary interventions in the catheter laboratory. Eur $J$ echocardiography 2009; 10(3): 341-349.

27. M L Rigby.Transoesophageal echocardiography during interventional cardiac catheterization in congenital heart disease. Heart 2001; 86 (90002): 1123-29. 
28. P Acar, Z Saliba, P Boenhoffer,D Sides, J Kachaar,Assessment of the geometrical profile of the Amplatzer and cardioseal septal occluders by tree dimensional echocardiography. Heart 2001; 85 (4): 451453.

29. P Beerbaum, H Corperich. H Edom, U Blanz, P Barth, J Hartman Atrial septal defect in pediatric patients: Noninvasive sizing with MR imaging.. Radiology 2003; 228 (2): 361-369.

30. Mogni G, Hijaji Z, Pandian N. Two or three dimensional transoesophageal echocardiography in patient selection and assessment of atrial septal defect closure by new DAS-Angel wings device. Circulation 1997; 96:17221728.

31. Ho S, McCarthy K, Rigby M. Anatomy of atrial and ventricular septal defects. J Interv Cardiol 2000; 13: 475-485.

32. Max G, Fulton D, Pandian n, Delineation of site, Relative size and dynamic geometry of atrial septal defect by real time three dimensional echocardiography. J Am Coll Cardiol 1995; 25: 482-490.

33. Dall'Agata A, McGhie J, Taams M, et al. Secundum atrial septal defect is a dynamic three-dimensional entity. Am heart J 1999; 137:1075-1081.

34. Ewert P, Berger F, Vogel M, et al. Morphology of perforated atrial septal aneurysm suitable for closure by transcatheter device placement. Heart 2000; 84:327331.

35. Hwang B, Lee P, Chiu P. Intracardiac ultrasound assessment of atrial septal defect: Comparison with transthoracic echocardiographic, angiocardiographic and balloon-sizing measurements. Cardiovasc Intervent Radiol 2001; 24:84.

36. Hijazi Z, Wang Z, Cao Q, et al. Transcatheter closure of atrial septal defects and patent foramen ovale under intracardiac echocardiographic guidance: feasibility and comparison with transoesophageal echocardiography. Catheter Cardiovasc Interv 2001; 52:194-199.

37. Lock J, Rome J, Davis R et al. Transcatheter closure of atrial septal defects. Circulation 1989; 79:1091-1099.

38. Zamora R, Lioyd T, Beekmann R, et al. Follow-up results of the multi-institutional phase 1 FDA supervised clinical trials of transcatheter occlusion of atrial septal defects with buttoned device. J Am Coll Cardiol 1997; 29:143A.

39. Charminati M, Hausdorf G, Tynan M, et al. Initial clinical experience of transcatheter closure of secundum atrial septal defect with a septal occlusion device. Eur Heart $J$ 1997;19 (suppl) :136.

40. Das G, Shrivastava S, O'Laughlin D, et al.. Intermediate term follow up of patients after percutaneous closure of atrial septal defects with the Das Angel wings device. Circulation 1996; 94: 1-56.

41. Rickers C, Hamm C, Stem H, et al. Percutaneous closure of secundum atrial septal defect with a new selfcentering device ("angel wings"). Heart 1998; 80:517521.
42. Lock J, Block R, Mckay R, et al. Transcatheter closure of ventricular septal defects. Circulation 1998; 78:361368.

43. Rigby M, Redington A. Primary transcatheter umbrella closure of perimembranous ventricular defect. $\mathrm{Br}$ Heart $J$ 1995; 73:368-371.

44. Lassuen P, Hansen D, Perry S, et. al. Transcatheter closure of ventricular septal defect, hemodynamic instability and anesthetic management. Anesth Analg 1995; 80:1075-1082,.

45. Sideris E. Walsh K. Haddad J, et al. Occlusion of congenital ventricular septal defect by the buttoned device. Heart 1997; 77:276-279,.

46. Thanopoulos B, Tsaousis G, Konstadopoulou G, et al. Transcatheter closure of muscular ventricular septal defect with the Amplatzer ventricular septal defect ocluder: initial clinical applications in children. $J \mathrm{Am}$ Coll Cardiol 1999; 33:1396-1399.

47. Spevak P, Bass J, Ben-sacher G, et al. Balloon angioplasty for severe congenital mitral stenosis. Am J. Cardiol 1990;66:472-476.

48. Grube E, Scheler G, Buellesseld L. Percutaneous aortic valve replacement for severe aortic stenosis in high risk patient using the second and third generation selfexpanding core valve prosthesis: device success and 30 day clinical outcome. J Am Col Cardiol 2007; 50 (1):6976 .

49. Foesbes Tj, Garekar S, Amin Z. Procedural results and acute complications in stenting native and recurrent coarctation of aorta is patients over 4 years of age: a Multi-institutional study. Cathet-Cardiovasc Interven 2007; 70 (2): 276-85.

50. Kessel-Schaefer, Migrating thrombus trapped in a patent foramen ovale. Circulation 2001; 103 (14): 1928.

51. Feldman T, Wasserman Hs, Hermann Hc et al. Percutaneous Mitral valve repair using edge-to-edge technique. 6 months results of the EVEREST phase 9 clinical trial. J Am Coll Cardiol 2005; 46:2134-40.

52. Feldman T, Leon MB. Prospects for percutaneous valve therapies. Circulation 2007;11:2866-77.

53. Feldman T. Percutaneous valve repair and replacement: challenges encountered, challenges met, challenges ahead. Circulation 2006; 113 (6):771-3.

54. Feldman T. Percutaneous Mitral annuloplasty: not analysis a cinch. Cathet Cardiovasc Interven 2007; 69: 1062-3.

55. St. Goar FG, James F1, Komtebedde J et al. Endovascular edge-to-edge Mitral valve repair. Shortterm results in a porcine Model. Circulation 2003; 108:1990-3.

56. Xino Jing Ma, Guo Ying Huang, Kue - Cun Liang, Zhong - Gen Chen Transoesophegeal echocardiography in monitoring, guiding and evaluating surgical repair of congenital cardiac malformation in children. Cardiology in Young 2007; 17: $301-306$. 